\title{
Review: heterocyclic antidepressants and rational psychological therapies are effective in older patients with mild to moderate depression
}

\author{
McCusker J, Cole M, Keller E, et al. Effectiveness of treatments of depression in older ambulatory patients. Arch Intern Med 1998 Apr \\ 13;158:705-12.
}

\section{Question}

In older ambulatory patients with depression, how effective are acute phase pharmacological and psychological treatments?

\section{Data sources}

Studies were identified by searching Medline (1981 to March 1995) using the subject headings depressive disorder, depression, aged, and clinical trials; and by searching PsycINFO (1984 to March 1995) using the subject headings major depression, aged, and therapy or treatment. Additional studies were identified by reviewing the bibliographies of articles and books.

\section{Study selection}

English or French language original studies were selected if they evaluated the effectiveness of acute phase pharmacological or psychological treatment using a prospective controlled study design in patients $\geqslant 55$ years of age with depression in an outpatient, community, or nursing home setting.

\section{Data extraction}

Data were extracted on sample size, mean depression rating scale scores at the beginning and end of treatment for each study group, standard deviations of the post treatment scores, and the $\mathrm{p}$ value for the after treatment comparison.

\section{Main results}

233 articles were identified of which 40 (37 different studies) met the selection criteria. There were 26 articles of pharmacological treatment and 14 articles of psychological treatments. 21 studies compared active drugs with placebo. In the 9 studies that compared heterocyclic drugs with placebo, heterocyclic drugs reduced the post treatment Hamilton Depression Rating Scale (HDRS) score (mean difference $-5.78,95 \%$ CI -8.31 to -3.25 , effect size -0.72 , CI -1.14 to -0.31 ). Other drugs compared with placebo showed smaller effects with some showing a statistically significant benefit (fluoxetine, trazodone, and phenelzine; HDRS differences $-2.40,-7.50$, and -0.75 , respectively). Overall, anti-anxiety drugs were not better than placebo. In the 17 studies that compared active drugs with active drugs, no differences existed between the treatment groups. Serotonin reuptake inhibitors appeared to be as effective as heterocyclic drugs. 12 studies compared psychological treatments with control treatments. Psychological treatments were classified as "rational" (cognitive or behaviourial) or "emotive" (supportive or dynamic). In the 4 studies that compared rational psychological treatment with an untreated control group, rational psychological treatment reduced the after treatment HDRS score (mean difference -7.25 , CI -10.10 to -4.40 , effect size -1.23 , CI -1.83 to -0.63$)$. This was not the case when rational treatments were compared with patients in a control group that received similar attention ( 2 studies, effect size -0.50 , CI -1.37 to 0.37 ) or emotive therapy ( 4 studies, effect size -0.35 , CI -0.93 to 0.22). None of the 4 studies comparing emotive treatment with an untreated control found a statistically significant benefit for emotive therapy.

\section{Conclusion}

In older ambulatory patients with mild to moderate depression, antidepressant drugs are more effective than placebo and rational psychological therapies are more effective than a no treatment control.

Source of funding: no external funding.

For correspondence: $\operatorname{Dr} J$ McCusker, Department of Clinical Epidemiology and Community Studies, St Mary's Hospital, 3830 Lacombe Avenue, Room 2508, Montreal, Quebec H3T 1M5, Canada. Fax +15147342652 .

Abstract and commentary also published in ACP Journal Club

\section{Commentary}

There are no high quality randomised, placebo controlled trials of drug or psychological therapy in the acute phase of old age depressive illness of whatever severity, and none could now be done for ethical reasons. ${ }^{1}$ Thus, a meta-analysis of this type is fraught with difficulty in dealing with such unsatisfactory raw material. Within these limitations this meta-analysis is sound, and the validity of its conclusions must ultimately be judged on the degree to which the studies analysed were methodologically flawed.

Firstly, elderly populations with depression are very heterogeneous. Samples that include outpatients, primary care patients, and people in residential or nursing homes are barely comparable; people in residential or nursing homes, for example, are likely to be much more physically ill than outpatients. The authors quite rightly conclude therefore that sample selection could account for the differences they observed.

Secondly, many studies were far too short (about 1 in 4 lasted only 4 weeks). The recommended length of time for a trial of antidepressant drugs at therapeutic dose in older patients is now deemed to be 6 weeks, ${ }^{2}$ and possibly longer.

Thirdly, the HDRS was the most commonly used outcome measure. The authors correctly state that the HDRS might have been unsatisfactory for less severely ill patients because of its heavy weighting on somatic items. In fact it is less suitable for any older depressed patients because non-depressed old people may score positively on these items.

The observation that "...attention alone may be an effective treatment" supports Blanchard's et al recent finding that such patients responded to simple intervention from a community nurse. ${ }^{3}$ The good practice point from this review is that time spent talking might be as effective as a drug.

Robin Jacoby, DM, FRCP, FRCPsych University of Oxford Oxford, UK

1 Anstey K, Brodaty H. Int J Geriatr Psychiatry 1995;10:265-79.

2 Bonner D, Howard R. Int J Geriatr Psychiatry 1995:10:259-64.

3 Blanchard M, Waterreus A, Mann A. Int J Geriatr Psychiatry 1995;10:289-98. 